\title{
Application of SVM and SVD technique based on EMD to the fault diagnosis of the rotating machinery
}

\author{
Junsheng Cheng ${ }^{\mathrm{a}, *}$, Dejie $\mathrm{Yu}^{\mathrm{a}}$, Jiashi Tang ${ }^{\mathrm{b}}$ and Yu Yang ${ }^{\mathrm{a}}$ \\ ${ }^{a}$ The State Key Laboratory of Advanced Design and Manufacturing for Vehicle Body, Hunan University, Changsha, \\ 410082, P.R. China \\ ${ }^{\mathrm{b}}$ College of Mechanics and Aerospace, Hunan University, Changsha, 410082, P.R. China
}

Received 7 December 2006

Revised 29 February 2008

\begin{abstract}
Targeting the characteristics that periodic impulses usually occur whilst the rotating machinery exhibits local faults and the limitations of singular value decomposition (SVD) techniques, the SVD technique based on empirical mode decomposition (EMD) is applied to the fault feature extraction of the rotating machinery vibration signals. The EMD method is used to decompose the vibration signal into a number of intrinsic mode functions (IMFs) by which the initial feature vector matrices could be formed automatically. By applying the SVD technique to the initial feature vector matrices, the singular values of matrices could be obtained, which could be used as the fault feature vectors of support vector machines (SVMs) classifier. The analysis results from the gear and roller bearing vibration signals show that the fault diagnosis method based on EMD, SVD and SVM can extract fault features effectively and classify working conditions and fault patterns of gears and roller bearings accurately even when the number of samples is small.
\end{abstract}

Keywords: Empirical mode decomposition, singular value decomposition, support vector machines, fault diagnosis

\section{Introduction}

The process of machinery fault diagnosis includes the acquisition of information, extracting feature and recognizing conditions. The later two are the priority.

Signal processing methods have been widely used to extract fault feature. Fourier transform (FT) which has been the dominating analysis tool for feature extraction of stationary signals could produce the statistical average characteristics over the entire duration of the data. However it fails to provide the whole and local features of the signal in time and frequency domain. However, the time-frequency analysis methods could generate both time and frequency information of a signal simultaneously. Therefore, in the most recent studies, the time-frequency analysis methods are widely used in fault feature extraction of rotating machinery vibration signal [1-3]. Among all available time-frequency analysis methods, the wavelet transform maybe the best one [4-6]. However, it still has some inevitable deficiencies. Firstly, energy leakage will occur when using wavelet transform to process signals due to the fact that wavelet transform is essentially an adjustable windowed Fourier transform. Secondly, the appropriate base function needs to be selected in advance. Moreover, once the decomposition scales are determined, the result of wavelet transform would be the signal under a certain frequency band. Therefore, wavelet transform is not a self-adaptive signal processing method in nature [7].

\footnotetext{
*Corresponding author. E-mail: signalp@ tom.com.
} 
Empirical mode decomposition (EMD, as defined in Section 2) is based on the local characteristic time scale of signal and can decompose the complicated signal into a number of intrinsic mode functions (IMFs, as defined in section 2) [8]. By analyzing each IMF that includes the local characteristic of the signal, the characteristic information of the original signal could be extracted more accurately and effectively. In addition, the frequency component included in each IMF not only relates to sampling frequency but also changes with the signal itself, therefore, EMD is a self-adaptive time frequency analysis method that is perfectly applicable to non-linear and non-stationary processing. Recently, Singular value decomposition (SVD, as defined in section 4) of matrix has been widely applied for regularization, noise reduction, signal estimation, signal detection, etc. [9]. Since the characteristics of periodic impulses usually occur whilst the rotating machinery exhibits local faults, SVD of matrix techniques also have been used to fault diagnosis of rotating machinery [10]. According to the matrix theory, singular value is the nature characteristic of matrix and owns favorable stability. Namely, when slight change of matrix element occurs, the variation of matrix singular value is small. However, before reconstructing phase space, reconstruction parameters, such as lag time and embedding dimension must be determined. Unfortunately, there is no mature theory to guide us to choose the reconstruction parameters [11]. Targeting the characteristics that periodic impulses usually occur whilst the rotating machinery exhibits local faults and the limitations of SVD techniques, the SVD technique based on EMD is applied to the rotating machinery fault diagnosis. In this proposed method, without requiring the choosing of reconstruction parameters with experience in advance, the IMFs resulted from EMD method are used to automatically form initial feature vector matrix, that is exactly the phase space. Furthermore, the singular values of the initial feature vector matrices could be obtained and used as the fault feature vectors of classifier.

Pattern recognition is another point of rotating machinery fault diagnosis. Conventional statistical pattern recognition methods and artificial neural networks (ANNs) classifiers are studied based on the premise that the sufficient samples are available, which cannot always be implemented in practice. Support vector machine (SVM, as defined in section 3) based on statistical learning theory which specializes in the smaller sample number question have better generalization than ANNs and guarantee that the local and global optimal solution are exactly the same. Meantime, SVM could solve the learning problem of a smaller number of samples [12]. Due to the fact that it is difficult to obtain sufficient fault samples in practice, SVM is introduced into the rotating machinery fault diagnosis in this paper due to its high accuracy and good generalization for a smaller sample number. The experimental results demonstrate the proposed diagnosis approach in which EMD, SVD and SVM are combined can be applied to the rotating machinery fault diagnosis efficiently.

\section{EMD method}

EMD method is developed from the simple assumption that any signal consists of different simple intrinsic modes of oscillations. Each linear or non-linear mode will have the same number of extrema and zero-crossings. There is only one extremum between successive zero-crossings. Each mode should be independent of the others. In this way, each signal could be decomposed into a number of intrinsic mode functions (IMFs), each of which must satisfy the following definition [8]:

1) In the whole data set, the number of extrema and the number of zero-crossings must either equal or differ at most by one.

2) At any point, the mean value of the envelope defined by local maxima and the envelope defined by the local minima is zero.

An IMF represents a simple oscillatory mode compared with the simple harmonic function. With the definition, any signal $x(t)$ can be decomposed as follows [8]:

1) Identify all the local extrema, then connect all the local maxima by a cubic spline line as the upper envelope.

2) Repeat the procedure for the local minima to produce the lower envelope. The upper and lower envelopes should cover all the data between them.

3 ) The mean of upper and low envelope value is designated as $m_{1}$, and the difference between the signal $x(t)$ and $m_{1}$ is the first component, $h_{1}$, i.e.

$$
x(t)-m_{1}=h_{1} .
$$




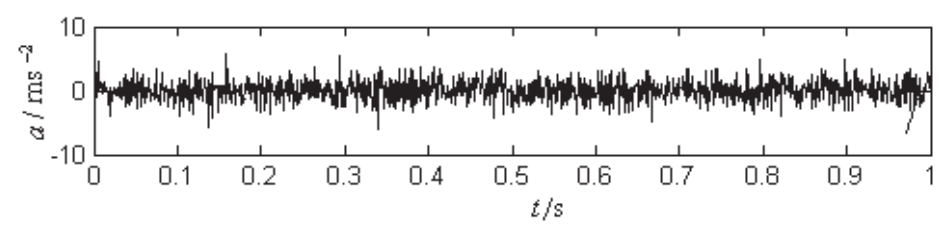

Fig. 1. The vibration acceleration signal of a normal gear.

Ideally, if $h_{1}$ is an IMF, then $h_{1}$ is the first component of $x(t)$.

4) If $h_{1}$ is not an IMF, $h_{1}$ is treated as the original signal and repeat 1), 2), 3); then

$$
h_{1}-m_{11}=h_{11}
$$

After repeated sifting, i.e. up to $k$ times, $h_{1 k}$ becomes an IMF, that is

$$
h_{1(k-1)}-m_{1 k}=h_{1 k}
$$

then, it is designated as

$$
c_{1}=h_{1 k},
$$

the first IMF component from the original data $c_{1}$ should contain the finest scale or the shortest period component of the signal.

5) Separate $c_{1}$ from $x(t)$, we could get:

$$
r_{1}=x(t)-c_{1}
$$

$r_{1}$ is treated as the original data and repeat the above processes; therefore, the second IMF component $c_{2}$ of $x(t)$ could be got. Let's repeat the process as described above for $n$ times, then $n$-IMFs of signal $x(t)$ could be got. Then,

$$
\left.\begin{array}{l}
r_{1}-c_{2}=r_{2} \\
\vdots \\
r_{n-1}-c_{n}=r_{n}
\end{array}\right\}
$$

The decomposition process can be stopped when $r_{n}$ becomes a monotonic function from which no more IMF can be extracted. By summing up Equations (5) and (6), we finally obtain

$$
x(t)=\sum_{j=1}^{n} c_{j}+r_{n}
$$

Residue $r_{n}$ is the mean trend of $x(t)$. The IMFs $c_{1}, c_{2}, \cdots, c_{n}$ include different frequencies ranging from high to low. The frequency components contained in each frequency band are different and they change with the variation of signal $x(t)$, while $r_{n}$ represents the central tendency of signal $x(t)$.

Figures 1, 2 and 3 show the vibration acceleration signal of the normal gear, gear with crack and gear with broken tooth, respectively. The decomposed results of vibration signal with broken tooth by using EMD are given in Fig. 4 that has 5 IMFs and 1 residue (In practice, the residue can be decomposed furthermore, but it makes no physical sense because the frequencies are too low). It can be seen from the figures that signal is decomposed into some IMFs with different time scales by which the characteristics of the signal can be presented in different resolution ratio.

\section{Support Vector Machine (SVM)}

Support Vector Machine (SVM) is developed from the optimal separation plane under linearly separable condition. Its basic principle can be illustrated in two-dimensional way as Fig. 5 [12]. Figure 5 shows the classification of a series of points for two different classes of data, class A (circles) and class B (stars). The SVM tries to place a linear boundary $H$ between the two classes and orients it in such way that the margin is maximized, namely, the distance 


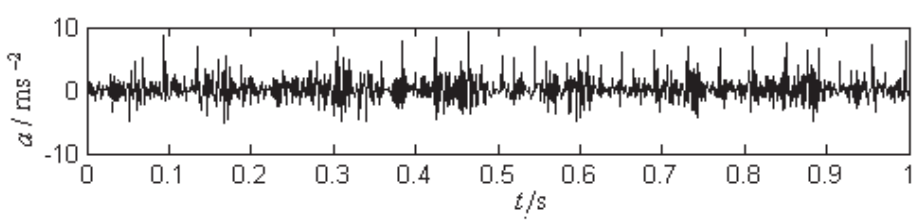

Fig. 2. The vibration acceleration signal of a gear with crack.

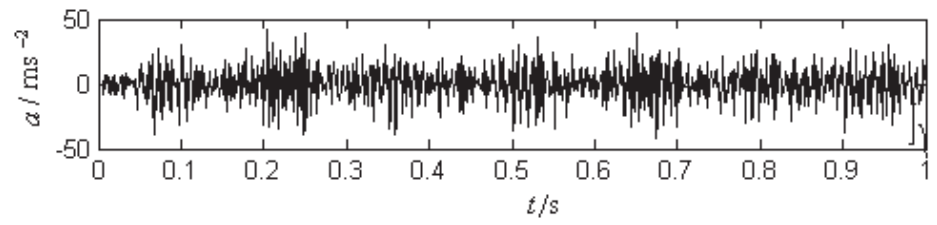

Fig. 3. The vibration acceleration signal of a gear with broken tooth.

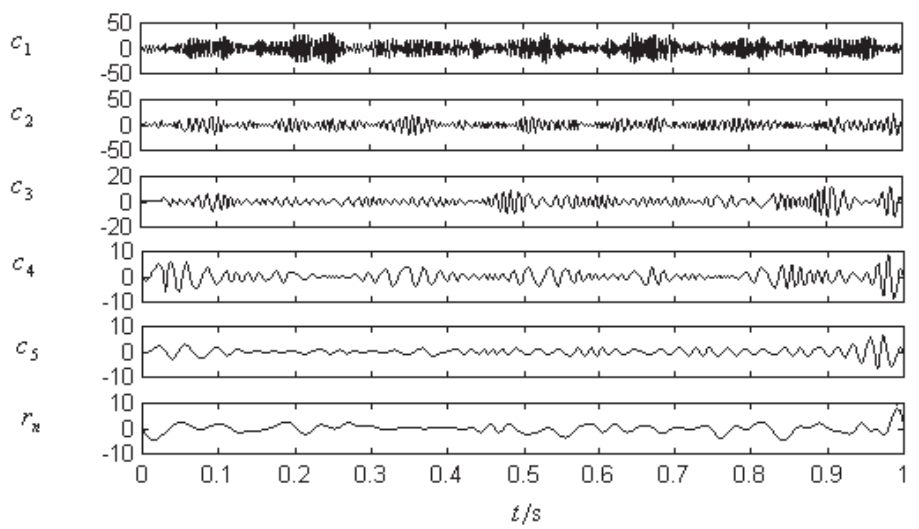

Fig. 4. The EMD decomposed results of vibration signal of the gear with broken tooth.

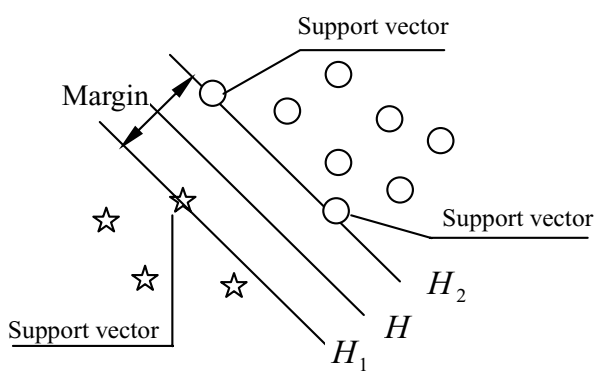

Fig. 5. Classification of data by SVM .

between the boundary and the nearest data point in each class is maximal. The nearest data points are used to define the margin and are known as support vectors.

Suppose there is a given training sample set $G=\left\{\left(x_{i}, y_{i}\right), i=1 \cdots l\right\}$, each sample $x_{i} \in R^{d}$ belongs to a class by $y \in\{+1,-1\}$. The boundary can be expressed as follows:

$$
\omega \cdot x+b=0
$$

where $\omega$ is a weight vector and $b$ is a bias. So the following decision function can be used to classify any data point 
in either class A or B:

$$
f(x)=\operatorname{sgn}(\omega \cdot x+b)
$$

The optimal hyperplane separating the data can be obtained as a solution to the following constrained optimization problem:

$$
\text { Minimize } \frac{1}{2}\|\omega\|^{2}
$$

$$
\text { Subject to } y_{i}\left[\left(\omega \cdot x_{i}\right)+b\right]-1 \geqslant 0, i=1, \cdots, l
$$

Introducing Lagrange multipliers $\alpha_{i} \geqslant 0$, the optimization problem can be rewritten as

$$
\text { Maximize } L(\omega, b, \alpha)=\sum_{i=1}^{l} \alpha_{i}-\frac{1}{2} \sum_{i, j=1}^{l} \alpha_{i} \alpha_{j} y_{i} y_{j}\left(x_{i} \cdot x_{j}\right)
$$

Subject to $\alpha_{i} \geqslant 0$

$$
\sum_{i=1}^{l} \alpha_{i} y_{i}=0
$$

The decision function can be obtained as follows

$$
f(x)=\operatorname{sgn}\left(\sum_{i=1}^{l} \alpha_{i} y_{i}\left(x_{i} \cdot x\right)+b\right)
$$

If the linear boundary in the input spaces is not enough to separate into two classes properly, it is possible to create a hyperplane that allows linear separation in the higher dimension. In SVM, it is achieved by using a transformation $\Phi(x)$ that maps the data from input space to feature space. If a kernel function

$$
K(x, y)=\Phi(x) \cdot \Phi(y)
$$

is introduced to perform the transformation, the basic form of SVM can be obtained

$$
f(x)=\operatorname{sgn}\left(\sum_{i=1}^{l} \alpha_{i} y_{i} K\left(x, x_{i}\right)+b\right)
$$

Among the kernel functions in common use are linear functions, polynomials functions, radial basis functions and sigmoid functions.

\section{The rotating machinery fault diagnosis method based on EMD, SVD and SVM}

If $A$ is a $N \times M$ real matrix, then there exists

$$
A=U \Lambda V^{T}
$$

where $U=\left[u_{1}, \cdots, u_{N}\right] \in R^{N \times N}, \quad U^{T} U=I ; V=\left[v_{1}, \cdots, v_{M}\right] \in R^{M \times M}, V^{T} V=I ; \Lambda \in R^{N \times M}, \Lambda=$ $\left[\operatorname{diag}\left\{\sigma_{1}, \cdots, \sigma_{p}\right\}: 0\right] ; p=\min (N, M) ; \sigma_{1} \geqslant \cdots \geqslant \sigma_{p} \geqslant 0$. The values of $\sigma_{i}$ are the singular values of matrix $A$ and the vectors $u_{i}$ and $v_{i}$ are respectively the $i$-th left and right singular vectors.

As we mentioned above, the decomposition results of EMD, IMFs, exactly contain various frequencies of the signal from high to low. Therefore, a division of frequency bands is formed automatically. Since this kind of decomposition is self-adaptive, the IMFs should reveal the nature characteristic of rotating machinery vibration signal more accurately. To avoid the difficulty of choosing the reconstruction parameters, all IMFs $\left(c_{1}, c_{2}, \cdots, c_{n}\right)$ are chosen to construct the initial feature vector matrices automatically. For convenience, all IMFs may be divided into two initial feature vector matrices $A$ and $B$ 


$$
A=\left[\begin{array}{l}
c_{1} \\
c_{2} \\
\vdots \\
c_{J}
\end{array}\right] \quad B=\left[\begin{array}{l}
c_{J+1} \\
c_{J+2} \\
\vdots \\
c_{n}
\end{array}\right]
$$

where $J=n / 2$ (when $n$ is even); $J=(n+1) / 2$ (when $n$ is odd number). Thus, the characteristic of vibration signal $x(t)$ could be revealed by the initial feature vector matrices $A$ and $B$. Furthermore, singular values of the initial feature vector matrices $A$ and $B$ could be obtained by applying SVD to initial feature vector matrices $A$ and $B$. For singular values can reflect the nature characteristic of matrix, characteristic of rotating machinery vibration signal can finally be described by singular values of the initial feature vector matrices. Therefore, singular values of initial feature vector matrices could be used as fault feature vectors. After a fault feature vector has been extracted, SVM could be chosen as classifier to identify the work condition and fault pattern of rotating machinery.

Taking the gear fault diagnosis for example, the gear fault diagnosis method based on EMD, SVD and SVM is given as follows:

1) Sample $N$ times at a certain sample frequency $f_{s}$ under the condition that the gear is normal, that the gear has the crack fault, broken teeth fault, respectively. And the $3 N$ signals are taken as samples that are divided into two subsets, the training samples and testing samples.

2) Each sample signal is decomposed by EMD method. Different signal has different amount that is denoted by $n_{1}, n_{2}, \cdots n_{3 N}$ of IMF components, and let $n=\max \left(n_{1}, n_{2}, \cdots, n_{3 N}\right)$. For some samples whose IMF components' amount $n_{k}$ is less than $n: n_{k}<n(k=1,2, \cdots, 3 N)$ it can be padded with zero to $n$ components: $c_{1}(t), c_{2}(t), \cdots, c_{n}(t)$, that is $c_{i}(t)=\{0\}\left(i=n_{k}+1, n_{k}+2, \cdots, n\right)$.

3) Construct initial feature vector matrices $A$ and $B$ to each gear vibration signal of each working condition according to Eq. (19). By applying SVD to the initial feature vector matrices $A$ and $B$, the singular value of initial feature vector matrices $\sigma_{A, j}, \sigma_{B, j}$ can be got

$$
\begin{aligned}
\sigma_{A, j} & =\left[\sigma_{A, j}^{1}, \sigma_{A, j}^{2}, \cdots, \sigma_{A, j}^{J}\right] \\
\sigma_{B, j} & =\left[\sigma_{B, j}^{J+1}, \sigma_{B, j}^{J+2}, \cdots, \sigma_{B, j}^{n}\right]
\end{aligned}
$$

where $\sigma_{A, j}^{1} \geqslant \sigma_{A, j}^{2} \geqslant \cdots \geqslant \sigma_{A, j}^{J}, \sigma_{B, j}^{J+1} \geqslant \sigma_{B, j}^{J+2} \geqslant \cdots \geqslant \sigma_{B, j}^{n}, j=1,2,3$ denotes the normal condition, condition with crack and condition with broken tooth, respectively.

4) Design SVM classifiers. The singular values of the initial feature vector matrices of the training samples are used as the fault feature vectors to be input to the SVM classifiers and the classifiers are trained. Actually the decision function $f(x)$ shown in Eq. (17) is determined only by the support vectors, so after it is obtained the fault feature vectors of testing samples can be input into the trained SVM classifier and then the working condition can be identified by the output of the SVMs classifier.

\section{Applications}

An experiment has been carried out on the small test rig shown in Fig. 6. The test rig is used for modeling different gear and roller bearing faults. The load's moment of inertia is $0.03 \mathrm{~kg} \cdot \mathrm{m}^{2}$. For convenience in narrating the analysis process, firstly we will discuss how to classify two work conditions of gears (normal and crack fault), that is, two-class problem. The fault is introduced by cutting slot with laser in the root of tooth, and the width of the slot is $0.15-0.25 \mathrm{~mm}$, as well as its depth is $0.1-0.3 \mathrm{~mm}$. The acceleration sensor has been fixed on the bearing house before 40 signals under two conditions are sampled with sample frequency of $1024 \mathrm{~Hz}$ and rotating frequency of $7 \mathrm{~Hz}$, among which five randomly chosen samples for each condition are taken as training samples, and the remaining are testing data.

Decompose the vibration signals of each group of the above two conditions with EMD method into a number of IMFs. It's noticed by analysis that the fault information of gears is mainly included in the first three IMF components. Therefore, the initial feature vector matrix $A$ only is established by the first three IMF components. Applying SVD 
Table 1

The identification results of proposed method for gear

\begin{tabular}{|c|c|c|c|c|c|c|c|c|}
\hline \multirow[t]{2}{*}{ Testing samples } & \multirow{2}{*}{\multicolumn{3}{|c|}{$\begin{array}{c}\text { Fault feature } \\
\text { Singular value } \sigma_{A, x}\end{array}$}} & \multicolumn{4}{|c|}{ SVM classifier } & \multirow{2}{*}{$\begin{array}{l}\text { Identification } \\
\text { results }\end{array}$} \\
\hline & & & & \multicolumn{2}{|c|}{$\begin{array}{c}\text { Distance } \\
\text { (10 training samples) }\end{array}$} & \multicolumn{2}{|c|}{$\begin{array}{c}\text { Distance } \\
\text { (6 training samples) }\end{array}$} & \\
\hline (1) Crack fault & 57.7139 & 29.6136 & 25.4805 & 2.0577 & $(+1)$ & 1.7603 & $(+1)$ & Crack fault \\
\hline (2) Crack fault & 65.9089 & 34.9811 & 19.3663 & 3.1017 & $(+1)$ & 2.7193 & $(+1)$ & Crack fault \\
\hline (3) Crack fault & 54.0604 & 30.3268 & 23.4564 & 1.4636 & $(+1)$ & 1.3412 & $(+1)$ & Crack fault \\
\hline (4) Normal & 33.3163 & 19.3193 & 9.9242 & -2.4079 & $(-1)$ & -1.6712 & $(-1)$ & Normal \\
\hline (5) Normal & 33.2064 & 19.2429 & 9.6941 & -2.4360 & $(-1)$ & -1.6916 & $(-1)$ & Normal \\
\hline (6) Normal & 43.2514 & 23.2916 & 15.4972 & -0.6295 & $(-1)$ & -0.3004 & $(-1)$ & Normal \\
\hline
\end{tabular}

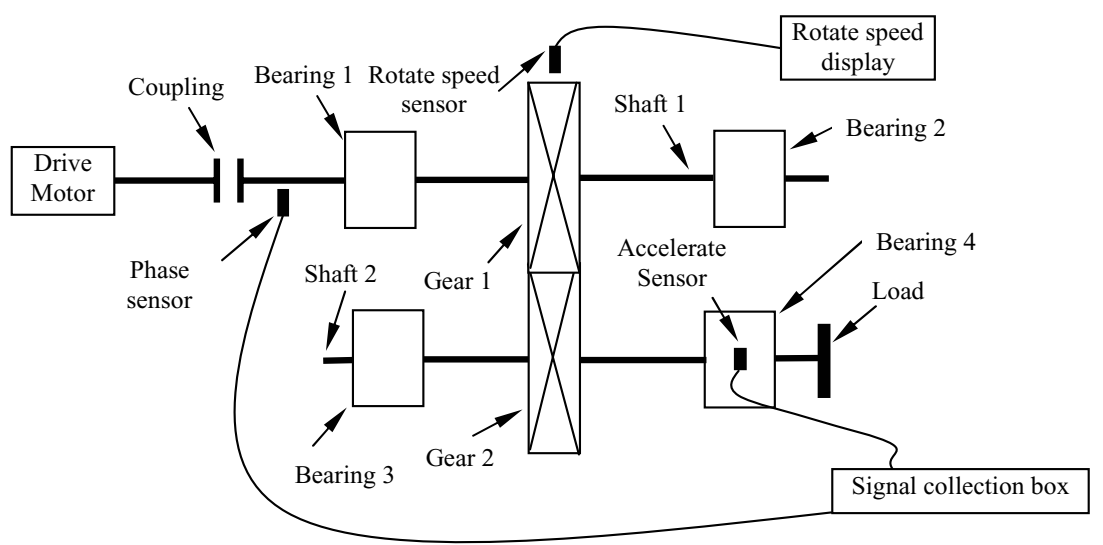

Fig. 6. Test rig.

to initial feature vector matrix $A$, the corresponding singular value $\sigma_{A, x}$ can be got, which could be regarded as the fault feature vectors to be input to the SVM classifier.

Define the normal condition of gear as $y=-1$ and the one with the crack fault as $y=+1$; choose the linear kernel function to calculate the decision function $f(x)$ and according to Eqs (10)-(14) we can obtain the parameters of SVM classifier, $\alpha=[0.0040,0,0.0076,0,0,0,0,0,0.0116,0]^{T},\|\omega\|=0.1523$ and $b=2.5485$. Then according to Eq. (17) the identification results of each testing sample are obtained, part of which are shown in Table 1. Obviously, the identification results are totally consistent with the fact. For further study of the application of SVM in the condition identification with smaller number of samples, the number of training samples decrease to six (half is normal condition and half is condition with crack fault) and the calculation procedure is the same as above. Here the parameters of the SVM classifier become $\alpha=[0.0070,0,0,0,0.0070,0]^{T},\|\omega\|=0.1183, b=-6.2941$. The identification results of the same testing samples are also shown in Table 1.

It can be seen from Table 1 that SVM classifier can still classify the two conditions of gears accurately after the training samples are decreased, which confirm fully that the SVM classifier can be applied successfully to the pattern recognition even in cases where only limited training samples are available. It also can be found, if we compare the distances between testing samples with different number of training samples and the optimal separating hyperplane $H$, that the distance decreases after the number of training samples become smaller although the gears can still be identified by SVM, which shows that in this way the whole performance of the classifier somewhat reduces.

Hereinbefore what we discuss is how to classify two conditions of gears (normal and crack fault), that is, two-class problem. When it comes to the multiple-class problems, that is, how to identify the gears with multiple-class faults (e.g. crack, broken teeth, etc.), generalizing method can be introduced to decompose the multiple-class problems into two-class problems which then can be trained with SVM. In other words, each time take one group of the training samples as one class and the rest, which do not belong to the former, can be taken as the other class. Hence for the $k(K \geqslant 3)$ classes' problems the classification of the input space can be achieved by $k$ decision-functions based on SVM [13]. 
Table 2

The identification results of two SVM classifiers for gear

\begin{tabular}{lrrrrl}
\hline Testing samples & \multicolumn{3}{c}{ SVM classifier } & Identification results \\
\cline { 2 - 4 } & \multicolumn{3}{c}{ Distance (SVM1) } & Distance (SVM2) & \\
\hline (1) Broken teeth & 1.1692 & $(+1)$ & & & Broken teeth \\
(2) Broken teeth & 1.0531 & $(+1)$ & & & Broken teeth \\
(3) Crack fault & -0.9828 & $(-1)$ & 2.7193 & $(+1)$ & Crack fault \\
(4) Crack fault & -1.0428 & $(-1)$ & 1.3412 & $(+1)$ & Crack fault \\
(5) Normal & -1.1905 & $(-1)$ & -1.6916 & $(-1)$ & Normal \\
(6) Normal & -1.1243 & $(-1)$ & -0.3004 & $(-1)$ & Normal \\
\hline
\end{tabular}

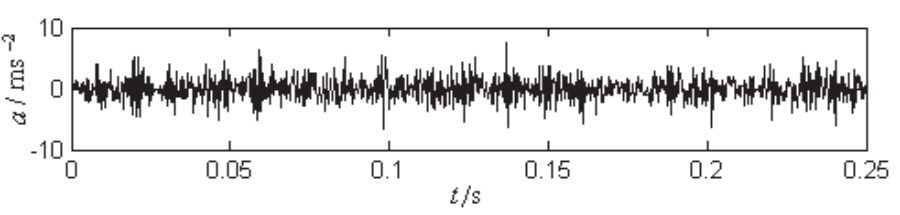

Fig. 7. The vibration acceleration signal of a normal roller bearing.

For example, two SVM classifiers are needed to design if three classes of gear conditions are to be identified, like normal, with crack fault and with broken teeth fault. First of all, for SVM1, define the condition with broken teeth fault as $y=+1$ and the other condition as $y=-1$, thus the broken teeth fault could be separated from other condition by SVM1. Then define the condition with crack fault as $y=+1$ and the other condition as $y=-1$ for SVM2, thus the crack fault could be separated from other condition by SVM2. Since we have known there are only three conditions to be identified, the remaining should be normal condition. The identification approach is the same as above, that is, extract 11 samples as training ones at random (3 samples with normal condition, 4 samples with crack fault and 4 samples with broken teeth fault). The part identification results are shown in Table 2 from which we can see that two SVM classifiers can identify the working conditions and fault patterns of gears accurately.

Similarly, above idea and diagnosis method could be applied to the roller bearing fault diagnosis. For fatigue of roller bearings may be occur on the inner-race $£$ <the outer-race, the rollers, or the cage, in this paper, an experiment has been carried out on the same experiment-rig with three 6311 roller bearings among which one is normal and the other two have inner-race and outer-race faults, respectively. Because of the limits of experiment condition, the cases that fault is on the rollers and the cage are not under consideration. The fault is caused by cutting slot with laser on its out-race or inner-race, and the depth is $0.13 \mathrm{~mm}$, as well as its width of the slot is $0.15 \mathrm{~mm}$ The experimental rotating frequency is $25 \mathrm{~Hz}$. The acceleration sensor has been fixed on the bearing seat before 40 signals under each mentioned-above circumstances are sampled with sample frequency of $4096 \mathrm{~Hz}$, among which 4 randomly chosen pieces for each condition are taken as training samples, and the remaining are testing data. Figures 7, 8 and 9 show the vibration acceleration signal of the normal roller bearing, roller bearing with out-race fault and roller bearing with inner-race fault, respectively. The decomposed results of roller bearing vibration signal with inner-race fault by using EMD are given in figure 10 that has 5 IMFs and 1 residue.

For SVM1, define the condition with inner-race fault as $y=+1$ and the other condition as $y=-1$, thus the inner-race fault could be separated from other condition by SVM1. Then define the condition with outer-race fault as $y=+1$ and the other condition as $y=-1$ for SVM2, thus the outer-race fault could be separated from other condition by SVM2. Since we have known there are only three conditions to be identified, the remaining is normal condition. If there exist roller fault, then the roller fault could be separated from other condition by SVM3 and so on. The analysis results show that the fault information of roller bearing is mainly included in the first five IMF components. Therefore, the initial feature vector matrix $A$ could be established by the first five IMF components. The singular value of initial feature vector matrix are regarded as fault feature vector and input to SVM classifiers. The part identification results are listed in Table 3. From Table 3 it is obvious that the identification results are coincide with real condition, which show that the fault diagnosis approach based on EMD, SVD and SVM can also identify the working conditions and fault patterns of roller bearings accurately even when the number of training samples is small. 
Table 3

The identification results of proposed method for roller bearing

\begin{tabular}{|c|c|c|c|c|c|c|c|c|}
\hline \multirow{3}{*}{$\begin{array}{l}\text { Testing } \\
\text { samples } \\
\text { (1) Inner-race fault }\end{array}$} & \multicolumn{3}{|c|}{$\begin{array}{l}\text { Fault feature vector } \\
\text { Singular value } \sigma_{A, x}\end{array}$} & \multicolumn{2}{|c|}{$\begin{array}{c}\text { SVM1 } \\
\text { Distance 1 } \\
\end{array}$} & \multicolumn{2}{|c|}{$\begin{array}{c}\text { SVM2 } \\
\text { Distnce 2 }\end{array}$} & \multirow{3}{*}{$\begin{array}{l}\text { Identification } \\
\text { results } \\
\text { Inner-race fault }\end{array}$} \\
\hline & 135.2059 & 95.9243 & 57.0451 & 0.5766 & $(+1)$ & & & \\
\hline & 37.9759 & 29.6915 & & & & & & \\
\hline \multirow[t]{2}{*}{ (2) Inner-race fault } & 130.9033 & 70.9121 & 55.0652 & 0.2893 & $(+1)$ & & & Inner-race fault \\
\hline & 38.1674 & 33.8395 & & & & & & \\
\hline \multirow[t]{2}{*}{ (3) Out-race fault } & 75.9796 & 28.4328 & 25.9605 & -1.0355 & $(-1)$ & 1.3568 & $(+1)$ & Out-race fault \\
\hline & 25.2359 & 23.0644 & & & & & & \\
\hline \multirow[t]{2}{*}{ (4) Out-race fault } & 77.5402 & 31.1577 & 21.6939 & -1.1818 & $(-1)$ & 1.4316 & $(+1)$ & Out-race fault \\
\hline & 15.4289 & 13.6663 & & & & & & \\
\hline \multirow[t]{2}{*}{ (5) Normal } & 49.8096 & 28.2896 & 19.7910 & -1.4674 & $(-1)$ & -1.4164 & $(-1)$ & Normal \\
\hline & 15.6391 & 14.1967 & & & & & & \\
\hline \multirow[t]{2}{*}{ (6) Normal } & 49.4454 & 29.5929 & 21.5680 & -1.4148 & $(-1)$ & -1.4191 & $(-1)$ & Normal \\
\hline & 18.0106 & 15.4145 & & & & & & \\
\hline
\end{tabular}

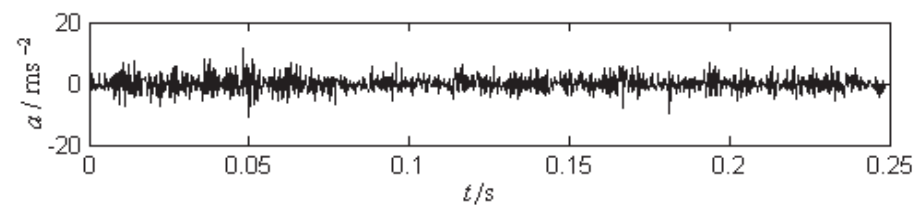

Fig. 8. The vibration acceleration signal of a roller bearing with out-race fault.

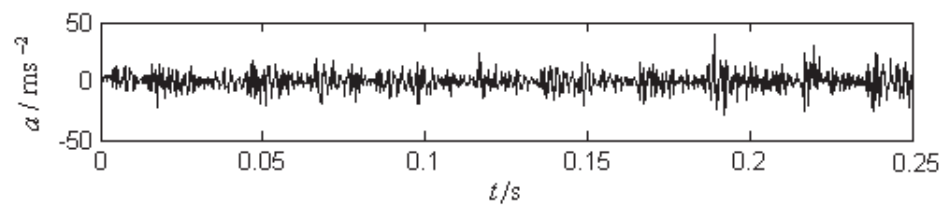

Fig. 9. The vibration acceleration signal of a roller bearing with inner-race fault.

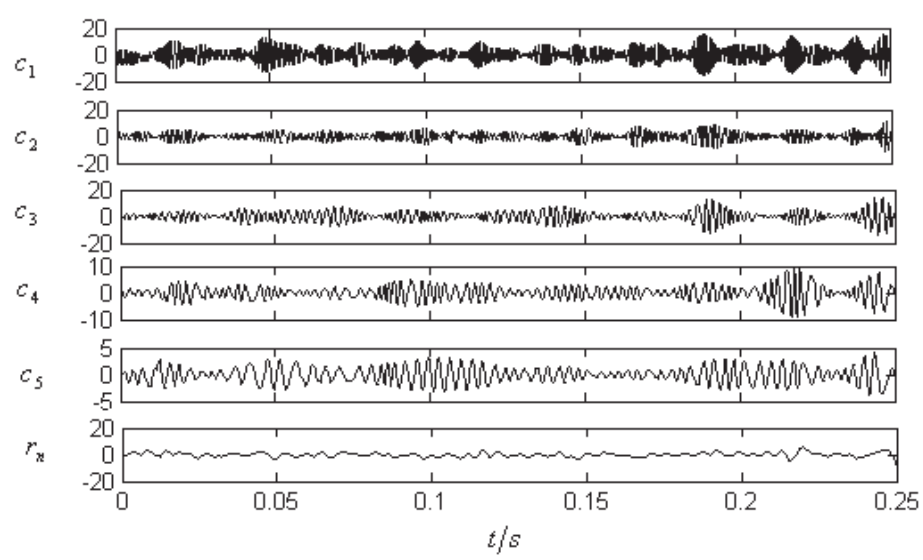

Fig. 10. The EMD decomposed results of vibration signal of the roller bearing with inner-race fault.

\section{Conclusion}

In SVD techniques, when lag time embedding techniques are used to reconstruct phase space, reconstruction parameters need to be chosen by experience in advance, which inevitably brings subjective influence to the accuracy of diagnosis results. EMD is a self-adaptive analysis method that can decompose the signal into a number of IMFs. 
By analyzing each IMF component that involves the local characteristic of vibration signal, the nature characteristic information of the original signal should be extracted more accurately and effectively. In this paper, the initial feature vector matrices could be formed automatically by some IMFs components that include main fault information. Therefore it is avoided to choose reconstruction parameters with experience. By applying the SVD to the initial feature vector matrices, the singular values of matrix could be obtained, which could be used as the fault feature vectors of classifier. In addition, targeting the limitations of the conventional statistical pattern recognition methods and ANNs classifies, SVM, which has better generalization than ANNs and can solve the learning problem of smaller number of samples quite well, has been introduced into the fault diagnosis of rotating machinery. It has been shown by the analysis results of gears and roller bearings that the fault diagnosis approach based on EMD, SVD and SVM can be applied to classify the working conditions and fault patterns of rotating machinery effectively and accurately even in case of smaller number of samples, which accordingly offers a new method for the fault diagnosis of rotating machinery.

What is necessary to point out is that due to the restriction of the experimental condition, it is inaccessible to etch a lesser slot on the faulted gears and roller bearings. Thus, the capability of this proposed method to identify different defect levels on a similar class fault still need to be researched further.

\section{Acknowledgment}

The support for this research under Chinese National Science Foundation Grant (No. 50775068) is gratefully acknowledged.

\section{References}

[1] J. Zou and J. Chen, A comparative study on time-frequency feature of cracked rotor by Wigner-Ville distribution and wavelet transform, Journal of Sound and Vibration 276(1) (2004), 1-11.

[2] N. Baydar and A. Ball, A comparative study of acoustic and vibration signals in detection of gear failures using Wigner-Ville distribution, Mechanical Systems and Signal Processing 15(6) (2001), 1091-1107.

[3] H. Oehlmann, D. Brie, M. Tomczak et al., A method for analyzing gearbox faults using time-frequency representations, Mechanical Systems and Signal Processing 11(4) (1997), 529-545.

[4] G. Meltzer, Nguyen Phong Dien. Fault diagnosis in gears operating under non-stationary rotational speed using polar wavelet amplitude maps, Mechanical Systems and Signal Processing 18(5) (2004), 985-992.

[5] Z.K. Peng and F.L. Chu, Application of the wavelet transforms in machine condition monitoring and fault diagnostics: a review with bibliography, Mechanical Systems and Signal Processing 18(2) (2004), 199-221.

[6] Z. Peng G, F. Chu and Y. He, Vibration signal analysis and feature extraction based on reassigned wavelet scalogram, Journal of Sound and Vibration 253(5) (2002), 1087-1100.

[7] H.T. Vicent, Damage detection using empirical mode decomposition method and a comparison with wavelet analysis. Proceedings of the Second International Workshop on Structural Health Monitoring, Stanford, 1998, 91-900.

[8] N.E. Huang, Z. Shen and S.R. Long, The empirical mode decomposition and the Hilbert spectrum for nonlinear and non-stationary time series analysis, Proceedings of the Royal Society of London Series 454 (1998), 903-995.

[9] A.J. Van Der Veen, E.F. Deprettere and A. Lee Swindlehurst, Subspace-based signal analysis using singular value decomposition, Proceedings of the IEEE 81 (1993), 1277-1308.

[10] J. Sandy, Monitoring and diagnostics for rolling element bearings, Journal of Sound and Vibration 22(6) (1988), 16-20.

[11] W.J. Wang and R.M. Lin, The application of pseudo-phase portrait in machine condition monitoring, Journal of Sound and Vibration 259(1) (2003), 1-16.

[12] V.N. Vapnik, The Nature of Statistical Learning Theory, New York: Springer-Verlag, 1995.

[13] Weston and C. Wetkins, Multi-class Support Vector Machine, London: Royal Holloway University of London, 1998. 

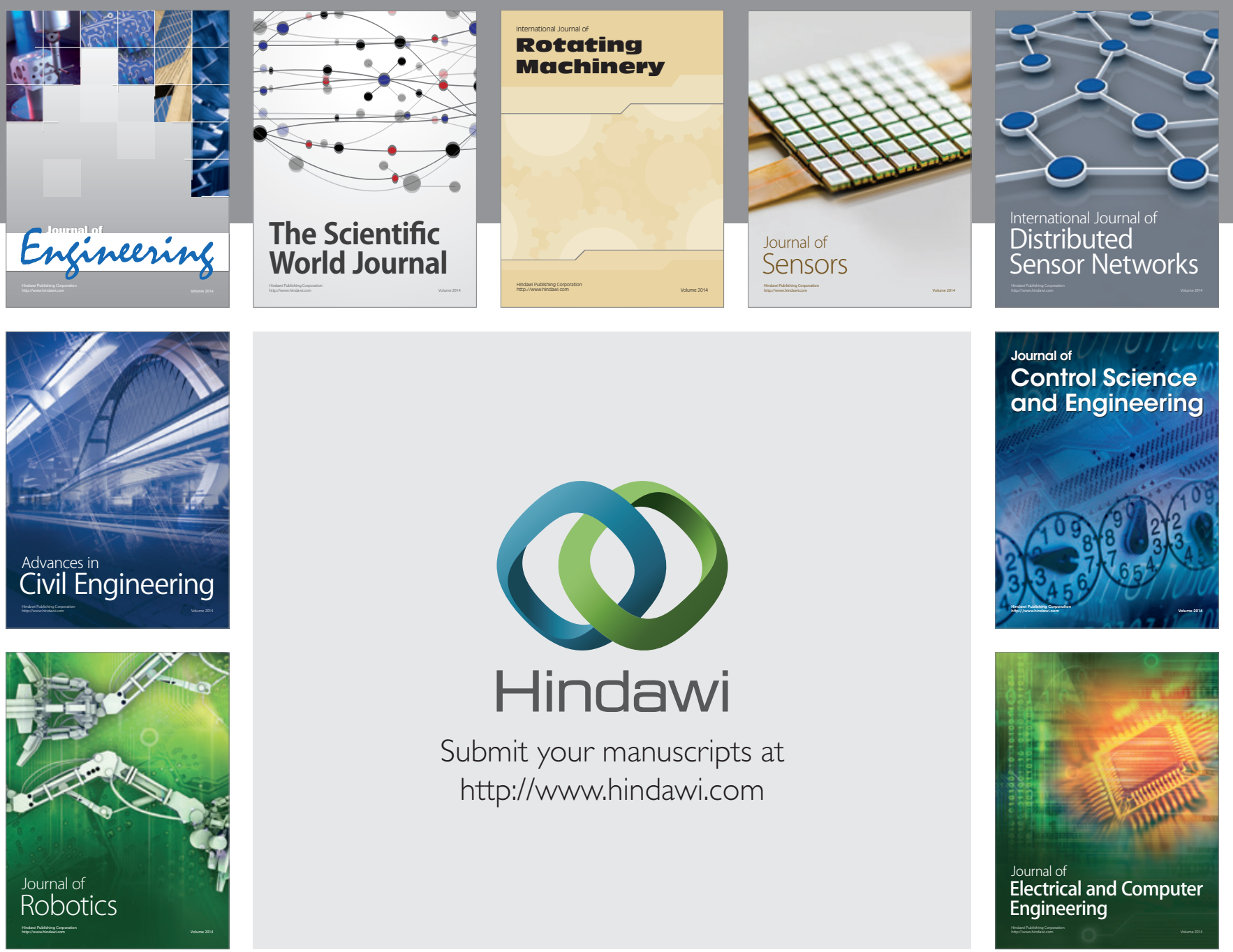

Submit your manuscripts at

http://www.hindawi.com
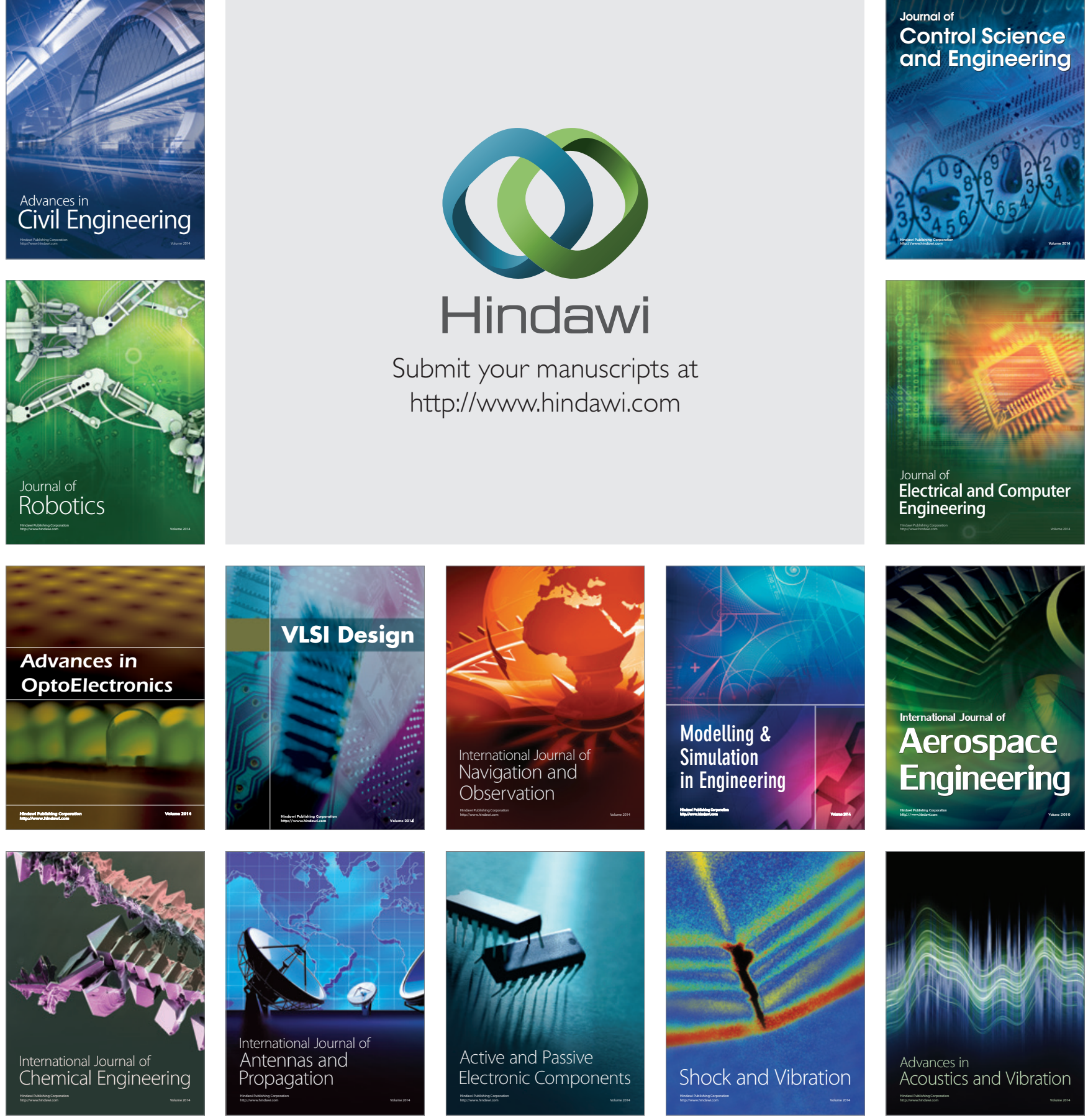